\title{
COVID-19 e Migrânea: 4 pacientes com migrânea sem aura e sua cefaleia na fase aguda da infecção
}

\section{Renata Gomes Londero}

Hospital de Clínicas de Porto Alegre, Porto Alegre, Rio Grande do Sul, Brasil

\section{Introdução}

O manejo da Cefaléia do Tipo Tensional (CTT), dentro de sua complexidade, inclui várias alternativas de tratamento. No Brasil, medicamentos analgésicos autoadministrados são frequentemente usados para interromper o mal-estar. Por outro lado, o uso, a difusão e o devido conhecimento de outros métodos e terapias não medicamentosas disponíveis tornaram-se essenciais para o alívio de quem sofre de dores.

\section{Objetivo}

Identificar métodos e terapias não farmacológicas utilizadas em publicações científicas para o alívio e tratamento da CTT.

\section{Método}

Estudo de revisão integrativa da literatura, abrangendo o período de 2000 a 2020, com base nas bases de dados bibliográficas PubMed, Science.gov, Web of Science e Portal Regional da BVS. Do volume inicial de 488 registros, após critérios de inclusão e exclusão, resultou 10 artigos originais para análise.

\section{Resultado}

O uso de estudos clínicos randomizados prevaleceu. Todos os estudos compuseram uma amostra com maior proporção de mulheres, exceto um. Todos os estudos mostraram resultados positivos de acordo com sua finalidade, revelando formas eficazes de aliviar ou tratar a CTT. Apenas um estudo não apresentou significância estatística.

\section{Conclusão}

Terapias como massagem, acupuntura e agulhas secas têm mostrado resultados promissores para intervenção na CTT. A combinação de intervenções e o uso de tecnologias inovadoras se destacaram.

Palavras-chave: Cefaleia do Tipo Tensional; Terapias Complementares; Tratamento Não Farmacológico 